\title{
Transverse mode dynamics in vertical cavity surface emitting lasers excited by fast electrical pulses
}

\author{
O. Buccafusca, ${ }^{\text {a) }}$ J. L. A. Chilla, J. J. Rocca, S. Feld, and C. Wilmsen \\ Center for Optoelectronic Computing Systems and Department of Electrical Engineering, \\ Colorado State University, Fort Collins, Colorado 80523 \\ V. Morozov \\ University of Colorado, Boulder, Colorado 80309 \\ R. Leibenguth \\ AT\&T Bell Laboratories, Breiningsville, Pennsylvania 18031
}

(Received 2 October 1995; accepted for publication 22 November 1995)

\begin{abstract}
We report measurements of the transient multimode dynamics of vertical cavity surface emitting lasers excited by short (100-200 ps rise times, 1 ns duration) electrical pulses. Fast changes on the spatial distribution of the output power and strong mode competition are observed. Numerical simulations show that the observed dynamics are due to the partial overlap of the different transverse modes through spatial hole burning. (c) 1996 American Institute of Physics. [S0003-6951(96)04505-0]
\end{abstract}

Recent advances in semiconductor device technology has produced two-dimensional arrays of vertical cavity surface emitting lasers (VCSELs), that, with modulation bandwidths of the order of $10-15 \mathrm{GHz},{ }^{1}$ are suitable for optical data links and optical signal processing. However, when semiconductor lasers are modulated at gigahertz frequencies, transient effects can affect their performance. In particular, the presence of higher order modes in the cavity can cause rapid variations of the spectral, spatial, and noise characteristics of the laser beam. Recent theoretical calculations ${ }^{2}$ predicted the importance of spatial hole burning effects in the dynamic evolution of transverse modes in VCSELs but, to our knowledge, no experimental studies on the subject have been reported. In this letter, we present spectrally and temporally resolved measurements of VCSELs under fast electrical excitation, which confirm the importance of mode competition and spatial hole burning in the dynamics in VCSELs, which are essentially different from the thoroughly studied dynamics in edge emitters. The transient response of edge emitters is the result of several longitudinal modes competing from the onset of laser operation. ${ }^{3}$ In contrast, the transient response measurements in VCSELs discussed here, show that the onset of a higher order spatial mode occurs after a delay dependent on prebias and pumping conditions. The presence of this higher order transverse mode is observed to produce a significant drop in the power of the $\mathrm{TEM}_{00}$ mode due to the redistribution of the available gain between the modes.

The lasers used in the measurements were gain guided GaAs/AlGaAs multiple quantum well VCSELs, with top and bottom mirrors consisting of step graded AlAs/AlGaAs distributed Bragg reflectors with 23 and 32 periods, respectively. ${ }^{4}$ The active region diameter was chosen to be $8 \mu \mathrm{m}$ to limit the number of transverse modes oscillating in the cav-

${ }^{a)}$ Electronic mail: osvaldo@lamar.colostate.edu ity. The VCSELs were prebiased through a bias tee and then electrically pumped using a fast pulse generator capable of providing square pulses of $0.8-1$ ns duration with 100-200 ps rise and fall times. Both the prebias and the peak currents were varied in order to characterize the VCSEL mode dynamics under different excitation conditions. Prebias currents were varied from $35 \%$ to $80 \%$ of the dc threshold value $\left(I_{t h}\right)$, while current pulse amplitudes ranging from 4 to 20 times $I_{t h}$ were added to pump the VCSEL. The pulsed currents were measured using a fast current probe (rise and fall times $<100 \mathrm{ps}$ ) constructed specifically for this study. The detection setup was similar to the one employed in Ref. 4. The output of the VCSEL was mixed in a nonlinear crystal with a reference pulse train of $\sim 100$ fs pulses from a Ti:sapphire laser, and the resulting sum-frequency signal was detected using a photomultiplier and standard locking techniques. The VCSEL was operated at a repetition rate of 80 $\mathrm{MHz}$ and the electrical pulses were synchronized with the optical pulses of the reference beam. The time resolution was limited by the jitter of the pulse generator to $<10$ ps. Spectral resolution was achieved by incorporating a monochromator of half-meter focal length to select the wavelength of the VCSEL output before up-conversion.

Figure 1 shows the spectrally and temporally resolved output of a VCSEL excited by $0.8 \mathrm{~ns}$ pulses with three different current pulse amplitudes and a prebias of $0.6 I_{t h}$. For this prebias current, pulses greater than 7 times $I_{t h}$ were necessary to achieve laser action within the duration of the electrical pulse. It is important to notice that for short excitation pulses, with durations of the order of the carrier lifetimes, the pulsed threshold currents are affected by the turn-on delay and therefore are larger than for dc operation. Also, in the excitation conditions used in our experiments (duty cycles $\sim 10 \%$ ), the intensity rolloff at high currents characteristic of VCSELs in dc operation ${ }^{5}$ was not observed since the temperature increase is less significant. For excitation pulses 
(a)
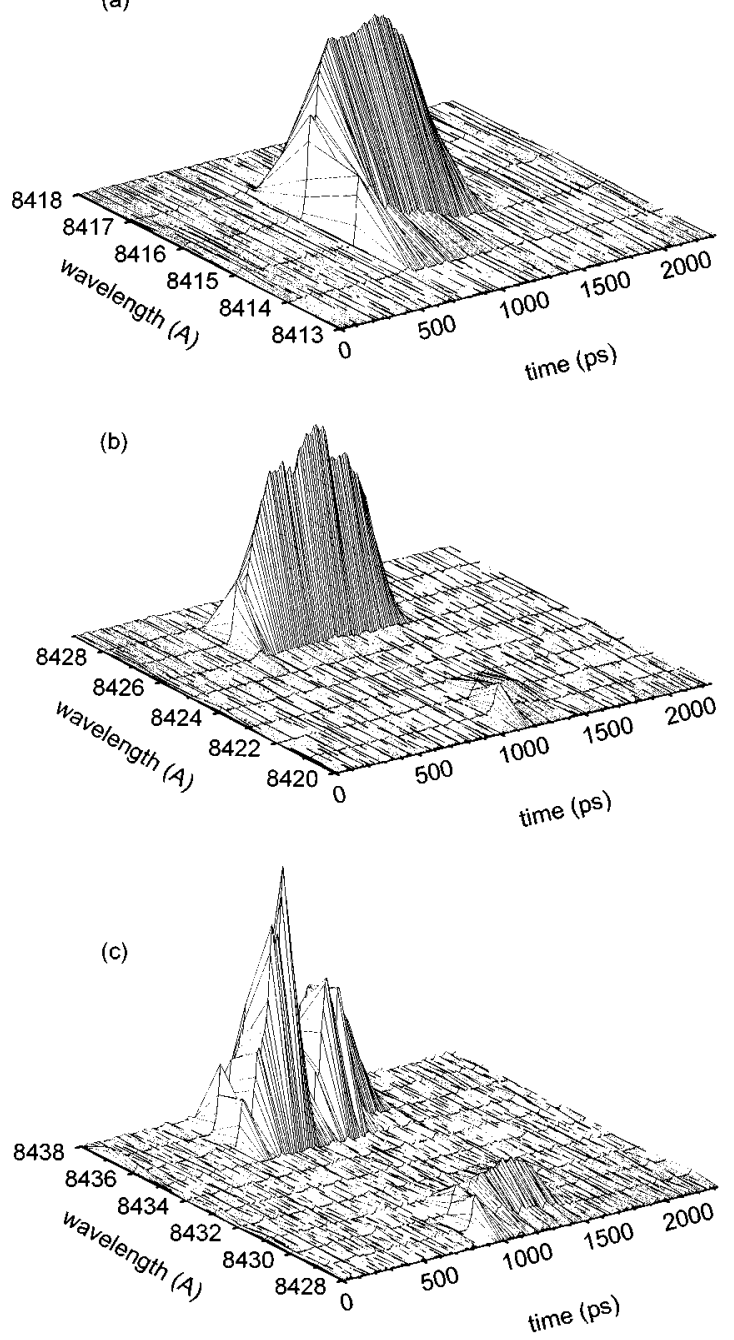

FIG. 1. Spectrally and temporally resolved output of a VCSEL prebiased at $0.6 I_{t h}$ and excited with three different pulse amplitudes: (a) $13 I_{t h}$, (b) 18 $I_{t h}$, and (c) $20 I_{t h}$. The observed change of the oscillation wavelength for different pumping conditions is due to thermal effects produced by the average excitation power.

with amplitudes below 15 times $I_{t h}$ [Fig. 1(a)] single mode operation is observed, as the gain is not large enough to overcome the losses of higher order modes. The output wavelength is observed to change rapidly during the rise of the optical pulse. A process which may account for this chirp is the dependence of the resonance wavelength of the FabryPerot cavity on carrier density. However, the resonance wavelength depends linearly on carrier density and should also produce wavelength variations during the relaxation oscillations; but these were not experimentally observed. A more likely cause of the observed chirp is self-phase modulation, which depends on the linewidth enhancement factor $(\alpha)$ and the derivative of the photon number. ${ }^{6}$ Using the published value of $\alpha \sim 4$ for similar VCSELs, ${ }^{7}$ the chirp due to this effect is evaluated to be of the order of $1.5 \AA$, in good agreement with the observed chirp. As the VCSEL is pumped harder, the evolution from single to multimode operation can be observed. For a current pulse of 18 times $I_{t h}$ [Fig. 1(b)] a second mode starts lasing after a delay of $\sim 460$
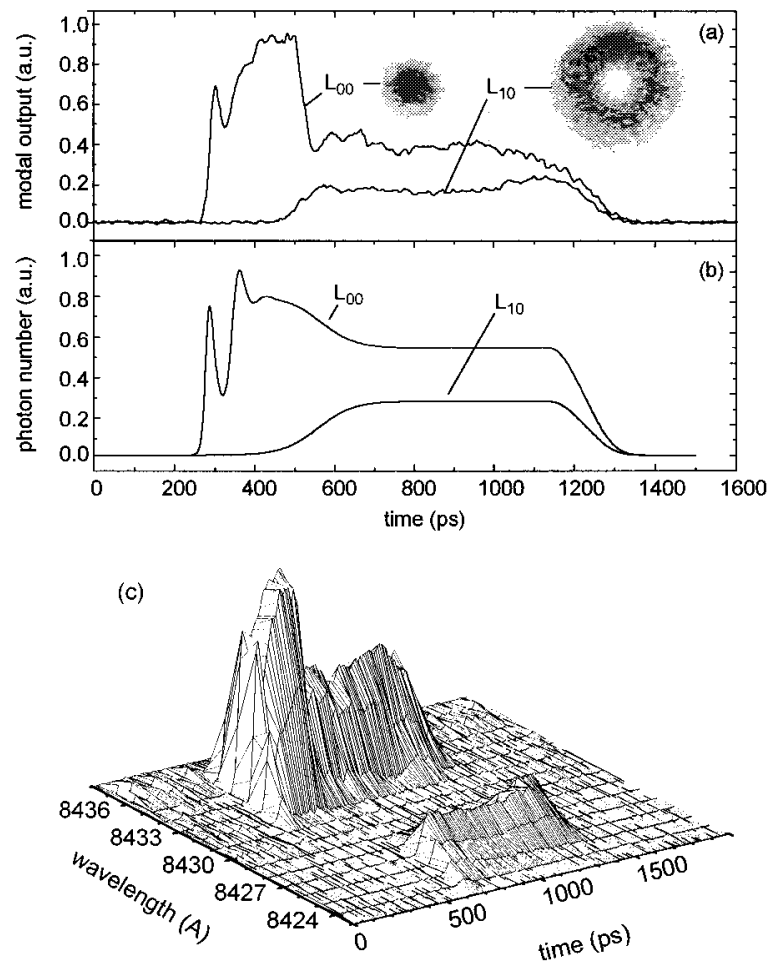

FIG. 2. (a) Spectrally integrated intensity of each mode in (c). The measured beam profiles are also shown. (b) Calculated photon number of each mode, (c) spectrally and temporally resolved output from a VCSEL showing multimode operation.

ps, while for a current pulse of 20 times $I_{t h}$ [Fig. 1(c)] the onset of the second mode occurs after a 300 ps delay. As the pumping currents were increased, the second mode was observed to lase with a stronger intensity after a shorter turn-on delay. However, different devices presented slightly different multimode dynamics, probably due to small variations in device parameters such as the active region diameter. For some devices it was possible to reach a situation in which the second mode became dominant and started lasing almost simultaneously with the first mode. When the prebias was increased to $0.8 I_{t h}$, current pulse amplitudes of only 3 times $I_{t h}$ were required by the VCSEL to lase within the electrical pulse and amplitudes of 13 times $I_{t h}$ were necessary to achieve multimode operation. We observed that during the $0.8-1$ ns pulse excitation used in this work, both polarizations coexist with approximately the same dynamics. This behavior is different from dc operation, where the polarization of the different transverse modes is usually well-defined.

The understanding of the transverse mode dynamics in VCSELs requires models that take into account the spatial distribution of carrier density and field amplitudes. A theoretical model, based on that described in Ref. 2 was used to simulate the observed multimode dynamics. The model considered two Laguerre-Gaussian modes $\left(L_{p l}\right)$ oscillating in the laser cavity: ${ }^{8}$ a Gaussian mode $L_{00}$, and a higher order symmetric mode $L_{10}$. The election of these modes was based on the observed symmetry of the far-field beam profile [see measured beam profiles in Fig. 2(a)]. Under this assumption, the problem has cylindrical symmetry and the carrier density 


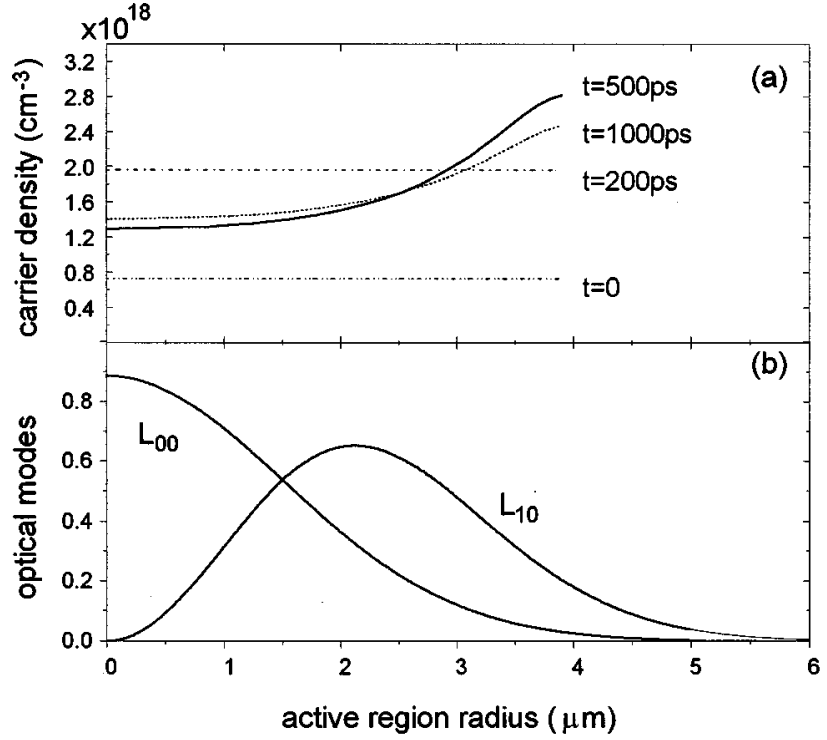

FIG. 3. (a) Calculated spatial distribution of the carrier density at different times, (b) computed intensity profiles of the optical modes.

in the active region depends only on the radial coordinate. To obtain a numerical solution, the active region was discretized in concentric subregions with equal volumes and the number of carriers in each subregion was calculated taking into account diffusion between neighboring zones. The calculations, which were performed assuming typical published values for material parameters ${ }^{9}$ reproduced the main features observed in the experiment.

Figures 2(a) and 2(b) show the measured and calculated evolution of the spectrally integrated intensity of each mode, for conditions (prebias $0.4 I_{t h}$, peak current $\sim 20 I_{t h}$ ) that result in the time resolved VCSEL spectrum shown in Fig. 2(c). Both the measurement and the calculation show that during the rise time of the electrical excitation, the $L_{00}$ mode starts lasing, showing highly damped relaxation oscillations (only the first peak is clearly noticeable in the experimental results). After $\sim 250 \mathrm{ps}$, the gain for a higher order mode overcomes the losses and the $L_{10}$ mode starts lasing. Notice that the onset of $L_{10}$ occurs after the relaxation oscillations of the first mode are already damped, which implies that although the modal photon number for $L_{00}$ reached its steady state, the carrier density is still changing in certain zones of the active region. The presence of a second mode in the cavity produces a significant drop in the intensity of the $L_{00}$ mode, which suggests that both modes are sharing the available gain. This competition between the two modes is a result of the overlap of the field distribution functions as shown by the theoretical calculations discussed next.

Figure 3(a) displays the evolution of the computed carrier density profile in the conditions of Fig. 2. Before laser action is achieved $(t=0,200 \mathrm{ps})$, the carrier density remains uniform, as the pumping current density is assumed uniform. The $L_{00}$ mode usually overcomes the modal losses first because the modal gain, which is determined by the overlap between the spatial distribution of carriers and the mode field profile, is higher for this mode. The computations show that the onset of the Gaussian mode $L_{00}$ produces a significant decrease of the carrier density in the center of the active region, where the optical mode has stronger intensity $(t=500$ ps). However, it has very little effect on the carrier density in the periphery of the active region, which continues to increase. After the onset of the $L_{10}$ mode $(t=1000 \mathrm{ps})$, the carrier distribution in the periphery is partially depleted. Hole burning in the region where both modes overlap significantly [2-4 $\mu \mathrm{m}$ in Fig. 3(b)] leads to a drop in the intensity of the $L_{00}$ mode which, in turn, causes a slight increase in the carrier density in the center of the active region. The simulations also showed that diffusion does not significantly affect the carrier distribution in the subnanosecond time scales of our study although, as pointed out in Ref. 2, it will play an important role in VCSELs excited by longer electrical pulses.

In summary, we performed spectrally and temporally resolved measurements of the output of electrically pumped VCSELs, which revealed the onset of a second order spatial mode with a delay dependent on pumping conditions. The onset of the second mode significantly reduces the intensity of the lowest order mode, an indication that the two modes compete for the same gain. This behavior is shown to be a result of the spatial distribution of carriers and the modal field profile in the laser cavity. This multimode dynamics is fundamentally different from the well-understood multimode dynamics in edge emitters, which present a strong competition among longitudinal modes from the beginning of laser action.

We acknowledge the contributions of P. Brusenbach and G. Vaschenko and we thank Dr. H. Temkin for lending the beam profiler. This work was supported by the Center for Optoelectronic Computer Systems through NSF Grant No. EEC-9015128 and by the Colorado Advanced Technology Institute Grant No. 0594-75-0738B.

${ }^{1}$ G. Shtengel, H. Temkin, P. Brusenbach, T. Uchida, M. Kim, C. Parsons, W. E. Quinn, and S. E. Swirhun, IEEE Photon. Technol. Lett. 5, 1359 (1993).

${ }^{2}$ A. Valle, J. Sarma, and K. A. Shore, IEEE J. Quantum Electron. 31, 1423 (1995).

${ }^{3}$ G. P. Agrawal and N. K. Dutta, Semiconductor Lasers, 2nd ed. (Van Nostrand Reinhold, New York, 1993).

${ }^{4}$ O. Buccafusca, J. L. A. Chilla, J. J. Rocca, C. Wilmsen, S. Feld, and R. Leibenguth, Appl. Phys. Lett. 67, 185 (1995).

${ }^{5}$ G. Hasnain, K. Tai, L. Yang, Y. H. Wang, R. J. Fischer, J. D. Wynn, B. Weir, N. K. Dutta, and A. Y. Cho, IEEE J. Quantum Electron. 27, 1377 (1991).

${ }^{6}$ T. L. Koch and R. A. Linke, Appl. Phys. Lett. 48, 613 (1986).

${ }^{7}$ D. Kuksenkov, S. Feld, C. Wilmsen, H. Temkin, S. Swirhun, and R. Leibenguth, Appl. Phys. Lett. 66, 277 (1995).

${ }^{8}$ K. Tai, Y. Lai, K. F. Huang, T. C. Huang, T. D. Lee, and C. C. Wu, Appl. Phys. Lett. 63, 2624 (1993).

${ }^{9}$ The material parameters used in the calculations were photon and carrier lifetimes of 6 and $800 \mathrm{ps,} \mathrm{respectively,} \mathrm{transparency} \mathrm{carrier} \mathrm{density} \mathrm{of}$ $10^{18} \mathrm{~cm}^{-3}$, material gain cross section of $1.9-10^{-16} \mathrm{~cm}^{2}$, spontaneous emission factor of $10^{-5}$, and ambipolar diffusion coefficient of 20 $\mathrm{cm}^{2} / \mathrm{s}$. 